\title{
Application of the New ISO 2631-5 to Health Hazard Assessment of Repeated Shocks in U.S. Army Vehicles
}

\author{
Nabih ALEM \\ U.S. Army Aeromedical Research Laboratory, Fort Rucker, Alabama 36362-0577, USA
}

Received February 4, 2005 and accepted March 18, 2005

\begin{abstract}
The U.S. Army Aeromedical Research Laboratory conducted a research program to develop a new methodology for health hazard assessment of tactical ground vehicle rides. This paper describes the new method and compares the health risk prediction using the new multiple shocks standard International Standards Organization (ISO 2631-5) to predictions by the current whole-body vibration (WBV) standard (ISO 2631-1). This article also describes the software tool developed to implement both parts of ISO 2631, as well as Army Regulation 40-10. The comparison shows the new standard to be more sensitive to cross-country terrain than other standards. Data analysis demonstrates the applicability of the new ISO 2631-5 standard to tactical ground vehicles, especially in the vertical axis.
\end{abstract}

Key words: Jolt, Health Hazard Assessment, Whole-Body Vibration, Vibration dose, Risk Assessment Code

\section{Introduction}

The health hazard assessment (HHA) is a program established by the U.S. Army with the objectives to prevent combat casualties, avoid performance decrements caused by routine operation of combat systems and reduce healthrelated deficiencies ${ }^{1}$. Repeated shock was identified as one of the potential hazards likely to be encountered during tactical ground vehicle (TGV) operations. To evaluate the safety of TGVs and their readiness for fielding, medical assessors and safety officers applied existing standards, such as International Standards Organization ISO 2631-1 standard $^{2)}$ and the British Standards Institute BS $6841^{3)}$. Unfortunately, those standards were developed primarily for civilian applications and not necessarily for military scenarios.

Field reports received in the mid 1980's by safety officers attributed adverse health effects to WBV exposure in military TGVs, even though these vehicles were judged to comply with existing WBV guidelines. According to one such anecdotal report, "hematuria was observed in $50 \%$ of the company" after completing a military exercise mission. Normally, these anecdotal reports would have triggered epidemiological surveys to document the extent of the problem and to establish any causal relationship between the medical symptoms and the ride characteristics. For various reasons, no such systematic surveys were conducted. However, the reports of medical symptoms were sufficiently credible to raise concerns over the validity of existing WBV standards. As a result of these concerns, the U.S. Army Aeromedical Research Laboratory (USAARL) set out to investigate the applicability of existing WBV standards to TGVs operating in military scenarios and to develop a militarily-relevant standard to use in evaluating occupant response to the repeated-shock environment that is commonly encountered in military vehicles. The USAARL then conducted a multi-year, multi-phase research program that culminated in the development of a new HHA method, the proposal and adoption of a new International Standards Organization (ISO) standard, and the development of a software tool that implements the complete methodology.

This article is based on a follow up study by the USAARL to gain initial experience with the new ISO standard. Results of the study also have been reported elsewhere ${ }^{4,5}$ and excerpts are included here with permission of the authors. As a background, it may be useful to describe briefly the original 
USAARL research program, most of which was performed under a contract with British Columbia Research Institute (BCRI). The experimental work was conducted at the USAARL multi-axis ride simulator (MARS) facilities in Fort Rucker, Alabama, and the data analysis and model development were completed at the BCRI facilities in Vancouver, British Columbia, Canada. A detailed account of the BCRI/USAARL research effort is given in a series of contractor reports by the BCRI team ${ }^{6-10}$. The following is a brief description of the research phases and a summary of the findings.

\section{Phase 1-Literature review ${ }^{6}$}

Over 1,200 published articles and reports were critically reviewed. Most of published studies agree that long-term exposure to vibration accelerates onset of lumbar spine disorders and possibly adversely affects the gastro-intestinal and cardiovascular systems. Few studies investigated human responses to repeated shock, but none investigated recovery. The review suggested potential approaches to development of an HHA method based on physiological, biochemical and biodynamic responses. Some biodynamic models, which range from single degree of freedom to three-dimensional and discrete parameter models, may have direct relevance to the development of a health hazard assessment method.

\section{Phase 2-Characterization of TGVs signatures ${ }^{7)}$}

WBV signatures from seven military vehicles, tested at Aberdeen Proving Ground, Maryland, were processed and characterized. Over 580 tri-axial acceleration signatures were collected from the M1A1 tank, M1A1 HTT, M1026 HMMWV, B109A3 self-propelled howitzer, M923A2 5-ton cargo truck, XM1076, and the M2HS Bradley fighting vehicle. A procedure was developed ${ }^{5)}$ to create generic motion signatures that simulated the shock and vibration environment of TGVs. Using this procedure, a motion signature was created mathematically to realistically simulate the motion environment of TGVs by synthesizing two signals: one to characterize the shocks and the other to characterize the near-continuous background vibration. This allowed the use of a handful of generic signatures to represent the majority of those that may be experienced in vehicles operating in military scenarios. These generic signatures were then used to drive the motion platform of MARS at Fort Rucker, Alabama, during subsequent experiments in Phases 3 and 4.

\section{Phase 3-Preliminary study ${ }^{8}$}

Ten subjects participated in pilot tests using the MARS facilities in Fort Rucker to determine the most sensitive human response measures to mechanical shock and repeated impact for use in the development of the experimental phase and in a dose-effect model. Spinal acceleration, internal pressure, chest and abdominal displacement measurements and electromyographic (EMG) activities showed similar frequency response patterns to the shocks applied at the seat and to the seat accelerations ${ }^{7}$. The similarity suggested seat acceleration might be used as the input parameter to the lumbar spine response models. The pilot study also recommended that such models should account for nonlinearity of response, differing horizontal and vertical inputs, and differing responses to positive and negative directions of shocks in the forward ( $\mathrm{x}$ ) and vertical ( $\mathrm{z}$ ) axes.

\section{Phase 4-Experimental Study ${ }^{91}$}

Fifty-four healthy, 19-40 year old U.S. Army Soldiers volunteered to participate in a series of motion exposures at the MARS, which simulated realistic WBV and repeated jolt scenarios likely to be encountered during TGV rides. Some of the tests were short duration and were designed to assess relative severity of shocks. In the long-duration experiments, subject fatigue and recovery were evaluated by exposing them to TGV ride signatures for up to $7 \mathrm{~h} / \mathrm{d}$, or $4 \mathrm{~h} / \mathrm{d}$ for $5 \mathrm{~d}$. The study concluded that biomechanical responses at the spine depended on shock axis, amplitude and direction, with the largest response resulting from vertical shocks. Subjective severity ratings to individual shocks were highly correlated with spinal acceleration. Subjects tolerated a 8-h vibration dose value, or $\operatorname{VDV}_{(8)}$, that exceeded the limit of 15 , recommended by the then-existing BS 6841. Some subjects were able to tolerate a $\mathrm{VDV}_{(8)}$ of 66 over a 7-h period, or a $\operatorname{VDV}_{(8)}$ of 60 over a 5-d period, without apparent health effects.

\section{Phase 5-Recommendations for a HHA method ${ }^{10,11)}$}

In the final phase of the USAARL research program, BCRI recommended that the HHA method incorporate: (a) biodynamic models to predict spinal acceleration, (b) regression models to predict peak compressive stress at the L4/L5 lumbar joint, (c) given peak acceleration, a fatiguebased model to quantify the cumulative effects of repeated shocks, and (d) an injury probability model that relates the cumulative dose to the probability of spinal injury within a normally-distributed population. Two biodynamic models were developed by BCRI. First, a single-degree-of-freedom (SDOF) model shown in Fig. 1 predicts response to horizontal shocks in the $\mathrm{X}$ and $\mathrm{Y}$ directions.

The other, a recurrent neural network (RNN) model shown 


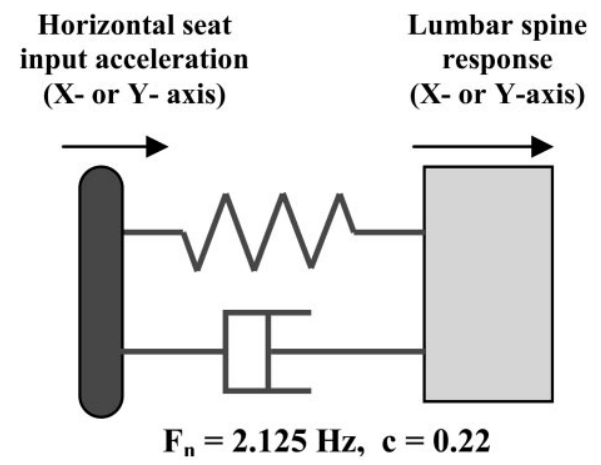

Fig. 1. Single degree-of-freedom model for prediction of lumbar spine kinematics response in the horizontal axes.

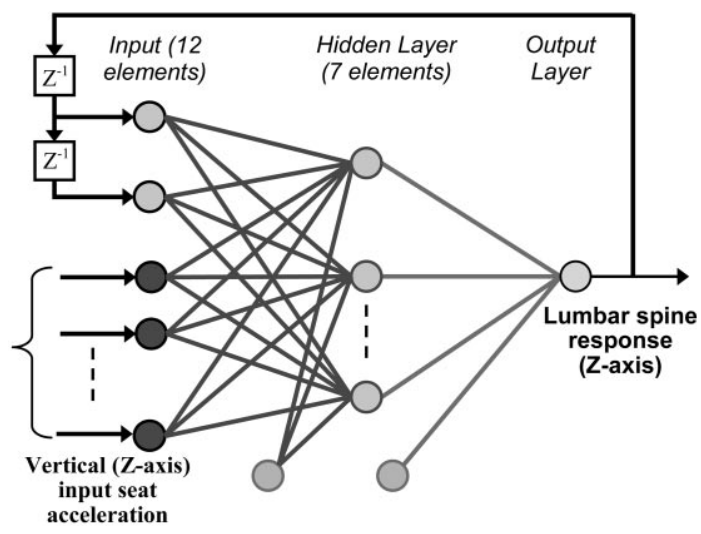

Fig. 2. Recurrent neural network model to predict lumbar spine response to multiple shocks in the vertical direction. Source: Morrison, et al. ${ }^{10)}$.

in Fig. 2, predicts the lumbar response to vertical shocks in the $+\mathrm{Z}$ direction. The constants for the two models were derived from the spinal response data that were generated in the experimental phase described above.

The biomedically-based BCRI approach for modeling and assessment of repeated shock formed the basis for proposing an amendment to the existing ISO 2631-1:1997. Meanwhile, a similar USAARL-led effort was underway in the U.S. to develop a standalone American National Standards Institute (ANSI) standard that incorporates the BCRI approach. Since the two on-going efforts followed the same modeling approach, the proposed ISO amendment and the draft ANSI standard were combined and a new draft international standard (DIS) emerged, which addressed specifically the repeated (or multiple) shocks. A new ISO standard, 2631$5^{12)}$, was approved in late 2003 and was published in 2004.

The new multiple shocks standard relies on the biodynamic models described above to calculate acceleration response at the lumbar spine. Once the spinal accelerations have been generated, an acceleration dose $\left(D_{x}, D_{y}, D_{z}\right)$ is calculated by summing peak acceleration responses that exceed certain thresholds for each axis. The dose then is normalized to an average workday based on duration of the available record and the expected length of the workday, to obtain $D_{x d}, D_{y d}$, $\mathrm{D}_{z \mathrm{~d}}$ and calculate the total daily exposure. Refer to the ISO 2631-5 document for full details of the calculations.

In addition, the new standard provides guidance for assessment of health affects of multiple shocks. Given the calculated total daily acceleration dose in each of the basicentric axes, they are combined to obtain an equivalent static stress compressive stress Sed. A daily equivalent static compression dose is then calculated and used to compute a risk factor, $\mathrm{R}$, for use in the assessment of the adverse health effects. For a typical career, the standard suggests that, $\mathrm{R}<$ 0.8 indicates a low probability of an adverse health effect and $\mathrm{R}>1.2$ indicates a high probability on an adverse health effect. This is equivalent to stating that $\mathrm{S}_{\mathrm{ed}}=0.5 \mathrm{MPa}$ and $\mathrm{S}_{\mathrm{ed}}=0.8 \mathrm{MPa}$ are the lower and upper boundary of a caution zone for a normal person during a typical working day ${ }^{10,12)}$. Again, the reader is referred to the ISO 2631-5:2004 document for details of the calculations.

The two boundary values of $S_{\text {ed }}$ (and R), which are "best estimates" derived from published vertebral strength data, should be validated against actual experience. Such validation studies usually take years to conduct. In order to gain early experience with the new multiple shock evaluation method, a comparative study was undertaken at the USAARL to compare the outcome of the new method of 2631-5 to that of two well-established shock evaluation methods specified in 2631-1. The remainder of this article describes this comparative study, discusses the application of the ISO parameters to the Army HHA requirement and briefly outlines a software tool that was developed to implements both parts of ISO 2631, as well as the formal HHA process.

\section{Materials and Methods}

\section{Data sources}

Ride pad accelerations were obtained from a dozen TGV's that were or are being developed by the U.S. Army. These included tracked and wheeled vehicles and water and land vehicles. All signatures were from seat cushion pads, that is, none of the data were from seat backs or from foot panels. Run conditions varied with terrain type, vehicle speed, and seat location. All vehicles were driven and occupied by healthy adult males who were seated upright. 


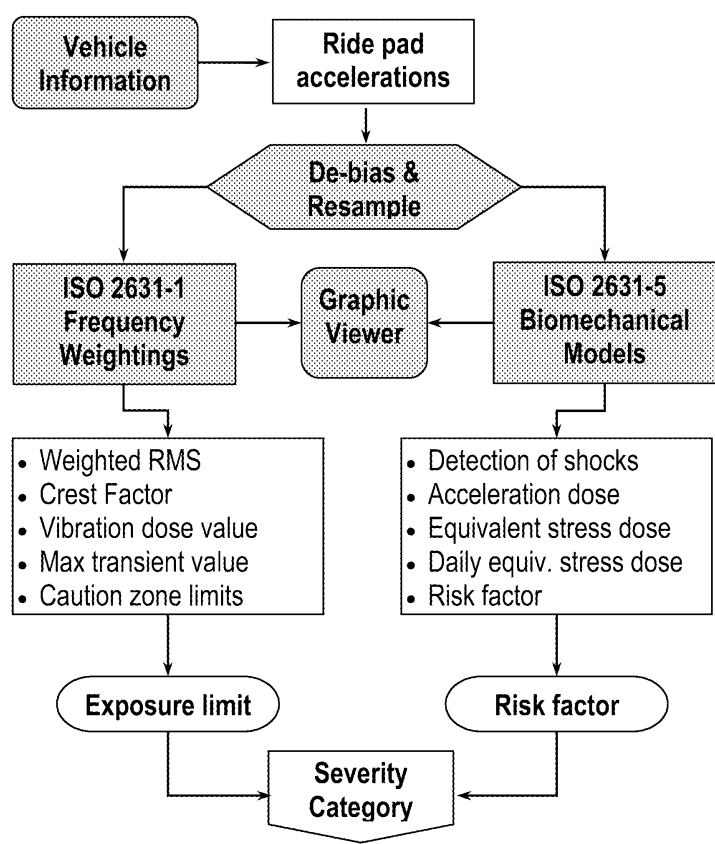

Fig. 3. Block diagram of the implementation of ISO 2631 standards (Part 1 and 5), resulting in assignment of hazard category to the tested vehicle.

\section{Data processing}

The data were stored in a standardized format that was developed by BCRI and USAARL to facilitate data exchange. A new software tool, described below, was developed and used to analyze all available signals to produce summaries that were saved in Microsoft Excel files. The summaries included, for each triaxial ride pad signature, the vehicle and seating information, which is replaced in this paper by a unique code. This should not detract from the goal of this paper, which is to compare repeated shock evaluation methods and not to evaluate the vehicles themselves. The terrain type and speed also was listed in the summaries to help explain any unusual outcome.

\section{WBV-JOLT Software}

A comprehensive software tool, WBV-JOLT ${ }^{13)}$, was developed to implement both Parts 1 and 5 of the ISO 2631 standard, and to extend the results of the ISO-based evaluation to the Army HHA process. One of the design goals of this software was to ensure its suitability for Army end-users, including industrial hygienists and occupational-health professionals. A user-friendly and stand-alone package then was developed, which required no external or complex operating environment. Although the new ISO 2631-5 provides a computer code for implementing the shock evaluation models, the code was written for the Matlab ${ }^{\circledast 13)}$ computing environment which may not be available to typical end users. Therefore, the data processing and model implementation were done in a combination of common highlevel languages (Visual Basic and Fortran) that do not use Matlab $^{\odot}$, and required the development of an efficient and accurate resampling module to resample the accelerations signals to 160 samples per second. The 160 samples/s is a requirement of the RNN model of vertical shock response model in 2631-5. A block diagram of the WBV-JOLT program is shown in Fig. 3.

\section{Evaluation methods}

Three evaluation methods were compared. The first method uses the total root-mean square of the weighted acceleration (also referred to here as WRMS) and described in ISO 2631-1:1997 as the basic evaluation method. The second method uses the vibration dose value (VDV) which is recommended in the 1997 standard as an additional method when the basic evaluation method is not sufficient to account for shocks that are embedded in the WBV signal. The mathematical definition of the WRMS, VDV and other evaluation indices are given in Annex A. The $\operatorname{VDV}_{(8)}$ is the value normalized to an 8 -h day, the average workday length. The third method is defined in the new ISO 2631-5:2004 standard and uses the equivalent static compression dose parameter to evaluate human response to multiple shocks. Here, we refer to this new method as the "Jolt method" because repeated jolt was the term used throughout the USAARL/BCRI research program to describe the rough rides encountered in tactical ground vehicles.

Normally, application of the Jolt method requires the calculation of the risk factor, $\mathrm{R}$, which takes into account the number of years and days per year of exposure and factors in the vertebral bone ultimate strength that depends on the age of the operator at the time of exposure. Since the other two methods, WRMS and VDV, do not incorporate lifetime exposure but can be normalized to an average (8-h) workday, the basis of comparisons in this paper was restricted to the $S_{\text {ed }}$ parameter, which is also parameter normalized to an average 8-h workday.

In addition to the three evaluation parameters WRMS, VDV and $S_{\text {ed }}$, two ratios described in Equations 7 and 8 in ISO 2631-1:1997 were computed and reported here. These two ratios, which are labeled Q7 and Q8, are supposed to trigger further evaluations when set thresholds are exceeded.

\section{The HHA Process}

The U.S. Army HHA program identifies more than a dozen health hazards, from shock and vibration to toxic gases, all 
Table 1. Convention used by the USAARL to assign health hazard severity categories of whole-body vibration and repeated shock

\begin{tabular}{|c|c|c|c|c|}
\hline \multicolumn{2}{|c|}{$\begin{array}{l}\text { Whole-Body Vibration } \\
\text { ISO 2631-1 }\end{array}$} & \multicolumn{2}{|c|}{$\begin{array}{l}\text { Repeated Shocks } \\
\text { ISO 2531-5 }\end{array}$} & \multirow{2}{*}{$\begin{array}{c}\text { HHA } \\
\text { AR 40-10 } \\
\text { Severity } \\
\text { Category }\end{array}$} \\
\hline $\begin{array}{l}\text { WBV Daily } \\
\text { Exposure Limit } \\
\text { (minutes) }\end{array}$ & $\begin{array}{l}\text { Vibration Dose } \\
\text { Value VDV } \\
\qquad\left(\mathrm{m} / \mathrm{s}^{1.75}\right)\end{array}$ & $\begin{array}{l}\text { Equivalent Daily } \\
\text { Stress, } S_{\text {ed }} \\
(\mathrm{MPa})\end{array}$ & Risk Factor, R & \\
\hline$<10$ & $>21$ & $>0.95$ & $>1.4$ & I-Catastrophic \\
\hline $10-30$ & $13-21$ & $0.65-0.95$ & $1.4-1.0$ & II-Critical \\
\hline $30-180$ & $4-13$ & $0.35-0.65$ & $1.0-0.6$ & III-Marginal \\
\hline$>180$ & $<4$ & $<0.35$ & $<0.6$ & IV-Negligible \\
\hline
\end{tabular}

For WBV, the daily exposure limit is the upper boundary of the caution zone defined in ISO 2631-1:1997. Assigning a severity category to the hazard is a necessary step in the HHA process prescribed in AR 40-10.

of which must be evaluated before fielding any new system ${ }^{13)}$. The evaluation process requires the medical assessor to classify the hazard according to its severity and probability of exposure. The severities of the WBV and jolt hazard are derived from the caution zone upper limit for WBV and from the equivalent daily stress $S_{\text {ed }}$ for repeated shock or the subsequently derived risk factor R. Since the guidance of ISO does not fall along the definitions used in AR 40-10, the upper limit of the caution zone and the $S_{\text {ed }}$ (and its dependent, the R-factor) were used for the four severity categories defined in the regulation. Table 1 lists the conventions used by the USAARL.

Based on these definitions, the severity categories are assigned automatically by the WBV-JOLT software, which allows the assessor to reassign the categories if necessary. To continue the HHA process, a hazard probability level must be assigned for the WBV and repeated shock exposure. HHA experts agree that these levels must be based on the mission profile of the item (or its fleet) and the frequency of its usage. At this time, there are no set guidelines for this process, so that one must judiciously evaluate the mission and assign a probability of exposure, perhaps in consultation with the item user. The Jolt software displays the language used in AR 40-10 to describe these probabilities, shown in Table 2, but does not automatically assign them. Instead, the user is required to manually enter the probability level for WBV and multiple shocks.

Finally, given the severity category and probability level of both WBV and repeated shock hazards, a risk assessment code (RAC) is assigned to the system that produced the hazard. The RAC is a numeric code (1-5) that is used by system developers and program managers to make acquisition and fielding decisions as prescribed by AR 40-10. The RAC is determined from the hazard severity category (1-4) and
Table 2. Whole-body vibration and repeated shocks hazard probabilities, as defined in AR 40-10

\begin{tabular}{cccc}
\hline \multicolumn{2}{c}{ Hazard Probability } & \multicolumn{2}{c}{ Likelihood of Occurrence } \\
Level & Label & in a vehicle & in the fleet \\
\hline A & Frequent & Frequently & Continuously \\
B & Probable & Several times & Frequently \\
C & Occasional & Sometime & Several times \\
D & Remote & Unlikely, possible & Unlikely, expected \\
E & Improbable & May never occur & Unlikely, possible \\
\hline
\end{tabular}

Assigning a probability level to the hazard is the second requirement of the health hazard assessment process prescribed by AR 40-10.

the hazard probability level (A-E), according to the lookup matrix shown in Table 3 . The entire HHA process is graphically shown in Fig. 4.

\section{Results}

Over 1,000 ride pad acceleration signatures were available for this study. All signals were processed using the WBVJolt software described earlier; however, a much smaller sample of signatures is included here. If the severity categories (SCs) derived from the WRMS and from the $S_{\text {ed }}$ for a given signature were both negligible (see definitions of Table 1), then it is reasonable to assume the signature was not likely to contain significant levels of repeated shocks, so that they would not be relevant to this study. In fact, over 90 percent of the examined signals fell into this negligible SC and were, therefore, excluded from further discussion.

There were about 70 runs whose WRMS-based SC was different from the $\mathrm{S}_{\text {ed }}$-based SC, indicating the presence of significant levels of shock. Nearly half of these tests had 
Table 3. Risk assessment codes provided in AR 40-10

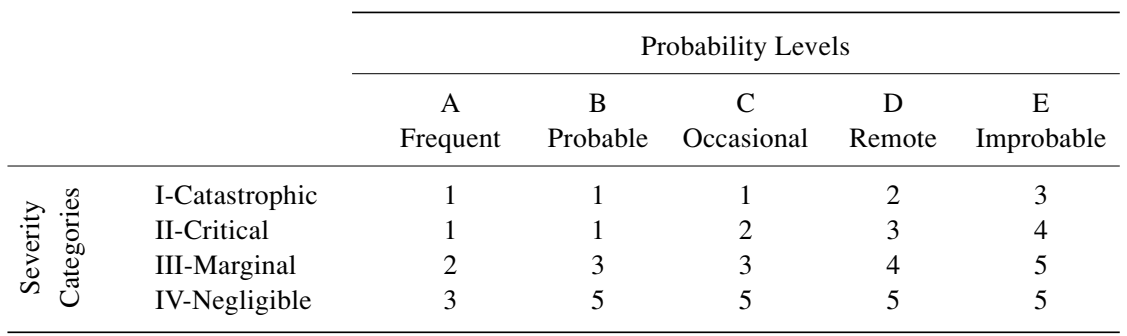

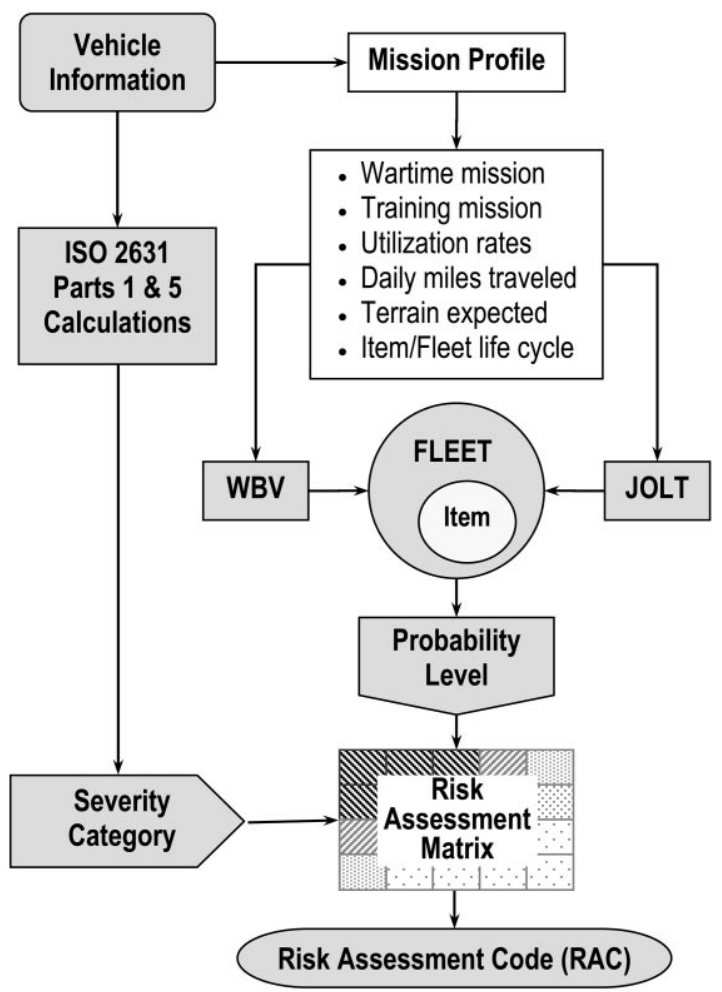

Fig. 4. Block diagram of the complete health hazard assessment methodology, as implemented by USAARL to meet Army Regulation 40-10 regulations.

The final outcome of the HHA process is a risk assessment code (RAC) that summarizes the safety of the vehicle for fielding.

comparable SCs to the other half and were, therefore, not selected for further discussion since they provided redundant information. The remaining 40 runs were deemed appropriate for this study. Visual inspection of these signals confirmed the presence of multiple shocks, as indicated by $\mathrm{SC}=1$ (catastrophic) or $\mathrm{SC}=2$ (critical). In rough rides that are now thought to be catastrophic or critical severity, the professional drivers testing the vehicles were heard on the voice channel of the taped data expressing their objections but no reports of adverse effects were filed. The terrain type and speed are included in the table to provide the reader with a better perspective on the results and to help explain unusual results. Although the vehicle type and seat location are likely to affect the vibration and shock signature, their identification is not necessary for the purpose of this discussion. Again, the purpose of this study was to compare different shock and vibration assessment methodologies and not to evaluate vehicles or seats. Detailed results of the calculations are provided in Table 2.

\section{Discussion}

The $\operatorname{VDV}_{(8)}$ and $S_{\text {ed }}$ parameters of interest are plotted against WRMS in Fig. 5. The use of WRMS as the abscissa was simply to scatter the two other variables and allow a better visual inspection of their values. Interestingly, the VDV $_{(8)}$ exhibited some correlation with WRMS. The ISO 2631-1 allows the use of an estimated vibration dose value (eVDV) and gives the values corresponding to the lower and upper boundaries of the caution zone as 8.5 (action level) and 17 (limit value), respectively (ISO 1997, clause B.3.1). The selected data set shows (Fig. 5, left plot) that the $\operatorname{VDV}_{(8)}$ did not exceed the action level of $8.5 \mathrm{~m} / \mathrm{s}^{1.75}$, and only one run exceeded the limit value of $17 \mathrm{~m} / \mathrm{s}^{1.75}$.

On the other hand, a plot of the $S_{\text {ed }}$ against the WRMS placed all but a few of the cases above the caution zone defined in ISO 2631-5 (Fig. 5, right plot), indicating that they may present a health risk. This was expected since the selection process of the sample of cases virtually eliminated all "benign" signatures as determined from the WRMS and the $S_{\text {ed }}$, and included most cases where the new method detected a high content of repeated shocks.

When the $\operatorname{VDV}_{(8)}$ is plotted against the $S_{\text {ed }}$, as shown in Fig. 7, a linear relationship $\left(\operatorname{VDV}_{(8)}=4.1 \times S_{\text {ed }}+1.5\right.$ with $\left.r^{2}=0.45\right)$ is suggested between the two parameters. Figure 7 also suggests an explanation why the VDV failed to detect signatures with high shock content. It appears that the threshold for $\operatorname{VDV}_{(8)}$, currently recommended in the ISO 2631-1, may be too high. In order for the VDV to be used for detecting high shock content in a vibration signature, 
the threshold should be lowered. If one accepts the correlation shown here, then the boundaries of a $\operatorname{VDV}_{(8)}$ caution zone should be $3.5 \mathrm{~m} / \mathrm{s}^{1.75}$ for the lower boundary (action level) and $4.8 \mathrm{~m} / \mathrm{s}^{1.75}$ for the upper boundary (limit value). With these reduced caution zone boundaries, the majority of the cases would be detected as requiring further analysis, but some would still go undetected.

The ISO 2631-1 standard (Clause 6.3.3) recommends the use of additional evaluation methods (beyond the basic RMS method) if the two screening ratios, Q7 and Q8, exceeded

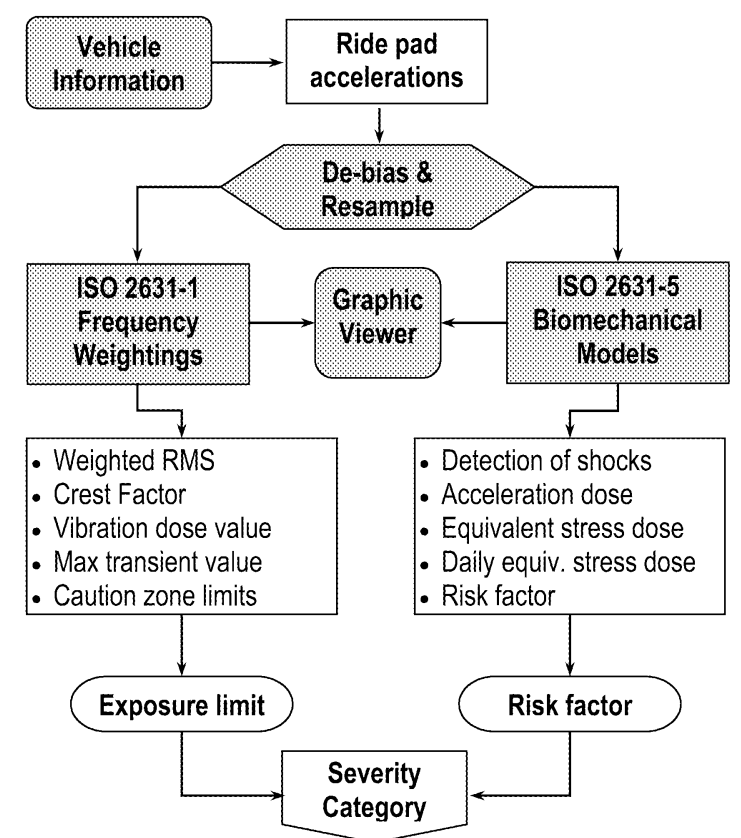

Fig. 5. Plots of the $S_{\text {ed }}$ and $\operatorname{VDV}_{(8)}$ against the WRMS, showing the recommended caution zones (horizontal bands) and action level (vertical dashed line).

Source: Alem, et al. ${ }^{4}$.
1.5 and 1.75 , respectively (Fig. 6). Both ratios are similar to the crest factor that was used unsuccessfully in the past to classify signals as ones containing shocks. The computed values of Q7 and Q8, given in Table 4, are plotted in Fig. 7. Again, the WRMS was used as a convenient mechanism to scatter the Q7 and Q8 values, which are the real focus of the plot. It is important to note that nearly all the selected tests produced Q7 ratios that were higher than the recommended threshold of 1.5, while nearly all Q8 ratios were below the threshold of 1.75 recommended as a flag for further analysis. The fact that $\mathrm{Q} 8$, which incorporates the VDV, does not trigger further analysis is consistent with the conclusion stated earlier in this section regarding the VDV. The Q7 ratio for most of the cases was able to indicate that additional analysis is warranted. In fact, all the Q7 ratios would have exceeded a threshold value of $\mathrm{Q} 7=1.25$. Therefore, it is suggested that trigger threshold for the Q7 ratio be reduced to 1.25 , and that $\mathrm{Q} 8$ not be used as a trigger for further evaluation of WBV signatures beyond the basic RMS method.

\section{Conclusions}

A new methodology to evaluate whole-body vibration containing multiple shocks has been developed. Unlike existing repeated shocks evaluation methods, such as the VDV method or the crest factor that are based on mathematical properties of a WBV signal, the new method is based on biomechanical response of the lumbar spine. Application of the new method is limited to seated healthy adult males. The methodology includes (a) the new ISO 2631-5 standard; (b) the WBV-Jolt software, and (c) the HHA extensions that implement AR 40-10.

The new method was compared to other methods (e.g.,
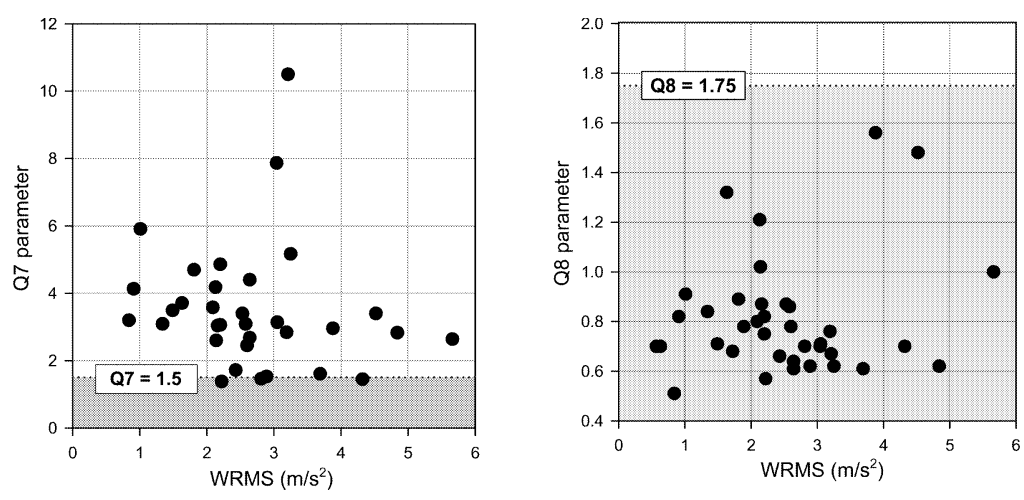

Fig. 6. Scatter plots of $Q 7$ and $Q 8$ screening ratios.

The green zones delineate values that would trigger the use of further evaluation methods, according to ISO 2531-1. Source: Alem, et al. ${ }^{4}$.

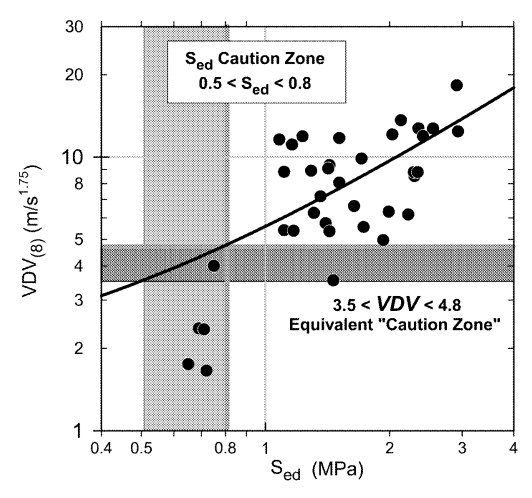

Fig. 7. Relationship between the $\operatorname{VDV}_{(8)}$ and the $S_{\text {ed }}$ parameters. 
the VDV) for the accurate detection of shocks in the WBV signature. Given a set of signals that were selected to have high shock content, the new method out-performed all other methods. This is not to say that the new method of ISO 2631-5 was able to predict injury from these signatures since these were test signatures and did not have injuries associated with them. The only way to verify the validity of the new method is prospective monitoring of the occupational health of vehicle operators over their careers. Although low- and high-risk thresholds were defined for the new method, these limits were based on the best biomechanical data available on lumbar spine vertebrae strength and failure. These threshold values should be monitored and, if necessary, revised based on credible new data that might be generated in the future.

\section{Acknowledgements}

The author wishes to thank Ernie Hiltz and Arlene BreauxSims for collecting and organizing the test data for this study,

Table 4. Results of data analysis for selected shock-rich ride pad signatures

\begin{tabular}{|c|c|c|c|c|c|c|c|c|c|c|}
\hline \multirow{2}{*}{$\begin{array}{c}\text { Terrain } \\
\text { Type }\end{array}$} & \multirow{2}{*}{\multicolumn{2}{|c|}{$\begin{array}{c}\text { Vehicle, Seat } \\
\text { Codes and } \\
\text { Speed }(\mathrm{km} / \mathrm{hr})\end{array}$}} & \multicolumn{2}{|c|}{ Ratios for Comparison } & \multicolumn{2}{|c|}{ Basic Method } & \multicolumn{2}{|c|}{ VDV Method } & \multicolumn{2}{|c|}{ Jolt Method } \\
\hline & & & $Q 7$ & $Q 8$ & $\underset{\left(\mathrm{m} / \mathrm{s}^{2}\right)}{\mathbf{W R M S}}$ & $\mathrm{SC}$ & $\underset{\left(\mathrm{m} / \mathrm{s}^{1.75}\right)}{\mathbf{V D V}_{(8)}}$ & $\mathrm{SC}$ & $\underset{(\mathrm{MPa})}{\mathbf{S}_{\mathrm{ed}}}$ & SC \\
\hline \multirow[t]{5}{*}{ Belgian Block } & V4-A & 40 & 1.72 & 0.66 & 2.43 & 3 & 8.83 & 3 & 1.11 & 1 \\
\hline & V4-E & 56 & 1.46 & 0.70 & 2.81 & 3 & 11.74 & 3 & 1.51 & 1 \\
\hline & V7-A & 32 & 1.53 & 0.62 & 2.89 & 3 & 9.08 & 3 & 1.42 & 1 \\
\hline & V4-E & 72 & 1.45 & 0.70 & 4.32 & 2 & 18.28 & 2 & 2.91 & 1 \\
\hline & V7-A & 40 & 1.57 & 0.64 & 4.78 & 2 & 15.96 & 2 & 5.20 & 1 \\
\hline \multirow[t]{5}{*}{ Washboard } & V2-G & 8 & 1.38 & 0.57 & 2.22 & 3 & 5.38 & 3 & 1.17 & 1 \\
\hline & V3-A & 16 & 2.68 & 0.61 & 2.64 & 3 & 8.91 & 3 & 1.29 & 1 \\
\hline & V3-D & 16 & 4.40 & 0.64 & 2.64 & 3 & 9.34 & 3 & 1.43 & 1 \\
\hline & V9-F & 40 & 5.17 & 0.62 & 3.25 & 3 & 11.11 & 3 & 1.16 & 1 \\
\hline & V3-A & 8 & 1.61 & 0.61 & 3.69 & 2 & 12.73 & 3 & 2.35 & 1 \\
\hline \multirow[t]{16}{*}{ Cross Country } & V5-A & 24 & 3.09 & 0.84 & 1.34 & 4 & 4.00 & 4 & 0.75 & 2 \\
\hline & V5-A & 24 & 3.71 & 1.32 & 1.63 & 3 & 5.55 & 3 & 1.73 & 1 \\
\hline & V3-D & 16 & 4.70 & 0.89 & 1.81 & 3 & 5.41 & 3 & 1.11 & 1 \\
\hline & V9-A & 16 & 3.58 & 0.80 & 2.09 & 3 & 5.73 & 3 & 1.40 & 1 \\
\hline & V5-A & 16 & 4.18 & 1.21 & 2.13 & 3 & 8.52 & 3 & 2.30 & 1 \\
\hline & V9-B & 32 & 2.60 & 1.02 & 2.14 & 3 & 8.81 & 3 & 2.29 & 1 \\
\hline & V2-G & 16 & 3.04 & 0.87 & 2.16 & 3 & 6.31 & 3 & 1.99 & 1 \\
\hline & V5-A & 40 & 4.86 & 0.82 & 2.20 & 3 & 5.35 & 3 & 1.43 & 1 \\
\hline & V9-F & 32 & 3.40 & 0.87 & 2.53 & 3 & 12.10 & 3 & 2.03 & 1 \\
\hline & V2-A & 16 & 3.09 & 0.86 & 2.58 & 3 & 8.07 & 3 & 1.51 & 1 \\
\hline & V5-A & 8 & 2.45 & 0.78 & 2.60 & 3 & 6.62 & 3 & 1.64 & 1 \\
\hline & V9-A & 24 & 3.14 & 0.71 & 3.05 & 3 & 8.81 & 3 & 2.34 & 1 \\
\hline & V3-C & 16 & 2.84 & 0.76 & 3.19 & 3 & 12.72 & 2 & 2.55 & 1 \\
\hline & V5-A & 32 & 2.96 & 1.56 & 3.88 & 2 & 9.87 & 3 & 1.71 & 1 \\
\hline & V5-A & 56 & 3.40 & 1.48 & 4.52 & 2 & 11.94 & 3 & 2.41 & 1 \\
\hline & V5-A & 48 & 2.64 & 1.00 & 5.66 & 2 & 13.64 & 2 & 2.13 & 1 \\
\hline \multirow[t]{6}{*}{ Paved Roads } & V3-A & 35 & 20.7 & 0.70 & 0.57 & 4 & 1.66 & 4 & 0.72 & 2 \\
\hline & V3-A & 32 & 19.4 & 0.70 & 0.63 & 4 & 1.75 & 4 & 0.65 & 2 \\
\hline & V3-C & 32 & 19.6 & 0.68 & 1.72 & 3 & 6.25 & 3 & 1.31 & 1 \\
\hline & V3-C & 35 & 26.9 & 0.78 & 1.89 & 3 & 7.19 & 3 & 1.36 & 1 \\
\hline & V3-C & 26 & 7.87 & 0.70 & 3.04 & 3 & 11.59 & 3 & 1.08 & 1 \\
\hline & V3-C & 19 & 10.5 & 0.67 & 3.21 & 3 & 11.91 & 3 & 1.23 & 1 \\
\hline \multirow[t]{5}{*}{ Secondary Roads } & W3-C & 29 & 4.13 & 0.82 & 0.91 & 4 & 2.35 & 4 & 0.71 & 2 \\
\hline & W3-C & 24 & 3.49 & 0.71 & 1.49 & 3 & 3.54 & 4 & 1.46 & 1 \\
\hline & W2-B & 11 & 3.06 & 0.75 & 2.20 & 3 & 6.16 & 3 & 2.22 & 1 \\
\hline & W3-C & 11 & 4.20 & 1.10 & 3.55 & 2 & 10.05 & 3 & 3.94 & 1 \\
\hline & W2-D & 40 & 2.83 & 0.62 & 4.84 & 2 & 12.42 & 3 & 2.93 & 1 \\
\hline \multirow[t]{3}{*}{ Water } & W1-D & - & 3.20 & 0.51 & 0.84 & 4 & 2.36 & 4 & 0.69 & 2 \\
\hline & W1-B & - & 5.91 & 0.91 & 1.01 & 4 & 4.97 & 3 & 1.93 & 1 \\
\hline & W1-A & - & 11.2 & 1.11 & 3.01 & 3 & 13.25 & 2 & 6.91 & 1 \\
\hline
\end{tabular}

The table provides a comparison between severity categories obtained with three different assessment methods. Source: Alem, et al ${ }^{4)}$. 
and Brad Bumgardner for expert computer programming, and the entire BCRI team who conducted the original research, developing the shock response models upon which the new method is built.

\section{Disclaimer}

The opinions, interpretations, conclusions, and recommendations are those of the author and not necessarily endorsed by the U.S. Army and/or the Department of Defense.

\section{References}

1) U.S. Army Regulation 40-10 (1992) Health hazard assessment program in support of the Army materiel acquisition decision process. Washington, D.C.: U.S. Department of the Army.

2) International Standards Organization (1997) Mechanical vibration and shock-Evaluation of human exposure to whole-body vibration-Part 1: General requirements. ISO 2631-1: 1997 standard.

3) British Standards Institute (1987) Guide to the measurement and evaluation of human exposure to whole-body mechanical vibration and repeated shock. BSI, London: BS 6841.

4) Alem N, Hiltz E, Breaux-Sims A, Bumgardner B (2004) Evaluation of new methodology for health hazard assessment of repeated shock in military tactical ground vehicles. Presented at NATO RTO-Applied vehicle technology symposium, Prague, Czech Republic. RTOAVT-110, Paper 7, 1-18.

5) Alem N, Hiltz E, Breaux-Sims A, Bumgardner B (2004) A new methodology for health hazard assessment of repeated shock in military tactical ground vehicles. Presented at the 24th Army Science Conference, Orlando, Florida. Poster, Paper No. KP-15.

6) Village J, Morrison J, Smith M, Roddan M, Rylands J, Robinson D, Brammer A, Cameron B (1995a) Development of a standard for the health hazard assessment of mechanical shock and repeated impact in army vehicles-Phase 1. Fort Rucker, AL: USAARL
Contract Report No. CR-95-1.

7) Roddan G, Brammer A, Village J, Morrison J, Smith M, Remedios B, Brown B (1995) Development of a standard for the health hazard assessment of mechanical shock and repeated impact in army vehicles-Phase 2. Fort Rucker, AL: USAARL Contract Report No. CR-95-2.

8) Village J, Morrison J, Robinson D, Roddan G, Nicol JJ, Springer MJN, Martin SH, Rylands J, Cameron B, Remedios B, Brown B (1995b) Development of a standard for the health hazard assessment of mechanical shock and repeated impact in army vehicles-Phase 3. Fort Rucker, AL: USAARL Contract Report No. CR-95-3.

9) Cameron B, Morrison J, Robinson D, Vukusic GA, Martin S, Roddan G, Albano JP (1996) Development of a standard for the health hazard assessment of mechanical shock and repeated impact in army vehicles-Phase 4. Fort Rucker, AL: USAARL Contract Report No. CR-96-1.

10) Morrison JB, Robinson DG, Roddan G, Rylands J, Cameron B, Remedios B, Brown B (1998) Development of a standard for the health hazard assessment of mechanical shock and repeated impact in army vehicles-Phase 5. Fort Rucker, AL: USAARL Contract Report No. CR-98-01.

11) Cameron B, Morrison J, Robinson D, Roddan G, Springer M (1998) Development of a standard for the health hazard assessment of mechanical shock and repeated impact in army vehicles final report Summary of Phases 1-5. Fort Rucker, AL: USAARL Contractor Report No. CR-98-02.

12) International Standards Organization (2004) Mechanical vibration and shock-Evaluation of human exposure to whole-body vibration-Part 5: Method for evaluation of vibration containing multiple shocks. ISO 2631-5: 2004(E).

13) Alem NM, Bumgardner BA (2005) WBV-Jolt 5.0 User's Manual, Fort Rucker, AL. USAARL Technical Report TR-2005-xx (in press). 


\section{ANNEX A}

Many of the WBV evaluation indices used in the body of the paper are defined in ISO 2631-1:1997 and 2631-5:2004 documents. For convenience of the reader, some of these definitions are repeated in this annex. Given the weighted acceleration $a_{w}(t)$, measured in $\mathrm{m} / \mathrm{s}^{2}$ as a function of time, and $T$ as the duration of the measurement in seconds, the weighted root-mean squared $(W R M S)$ is defined as:

$$
W R M S=\left[\frac{1}{T} \int_{0}^{T} a_{w}^{2}(t) d t\right]^{1 / 2}
$$

The running RMS method that takes into account occasional shocks by use of a short integration time constant, $\tau$. In this method, the WBV magnitude is evaluated using the Maximum Transient Vibration Value (MTVV), given as the maximum in time of $a_{w}\left(t_{0}\right)$, defined by:

$$
M T V V=\max \left[\frac{1}{\tau} \int_{t_{0}-\tau}^{t_{0}} a_{w}^{2}(t) d t\right]^{1 / 2}
$$

The vibration dose value is defined in $\mathrm{m} / \mathrm{s}^{1.75}$ as

$$
V D V=\left[\frac{1}{T} \int_{0}^{T} a_{w}^{4}(t) d t\right]^{1 / 4}
$$

The following two screening ratios are defined in ISO 2631-1 as equations 7 and 8 .

$$
\begin{aligned}
& Q 7=\frac{M T V V}{W R M S} \\
& Q 8=\frac{V D V}{W R M S \cdot T^{1 / 4}}
\end{aligned}
$$

The acceleration dose is defined in ISO 2631-5 as the cumulative function of peak magnitudes in a recorded signal, or

$$
D_{k}=\left[\sum_{i=1}^{m} A_{i k}^{6}\right]^{1 / 6}
$$

where $A_{i k}$ is the $i$-th peak of the acceleration in the $k$-direction $(k=x, y, z)$, and $m$ is the number of peaks in the measured signal. The average daily acceleration dose for each direction $k$ is obtained by normalizing the dose measured over a period $t_{m}$, to the duration of an average workday $t_{d}$,

$$
D_{k d}=D_{k}\left[\frac{t_{d}}{t_{m}}\right]^{1 / 6}
$$

The equivalent daily stress is defined as:

$$
S_{e d}=\left[\sum_{k=x, y, z}\left(m_{k} D_{k d}\right)^{6}\right]^{1 / 6}
$$

where $m_{k}$ are empirical constants for $\mathrm{x}, \mathrm{y}$, and $\mathrm{z}$ directions. Recommended values for these constants are: $m_{x},=0.015$, $m_{y}=0.035$ and $m_{z}=0,032 \mathrm{MPa} /\left(\mathrm{m} / \mathrm{s}^{2}\right)$. Finally, the health risk for healthy seated males may be projected to a lifetime, using a risk factor $R$ defined as:

$$
R=\left[\sum_{i=1}^{n}\left(\frac{S_{e d} \cdot \text { days }^{1 / 6}}{S_{u i}-c}\right)^{6}\right]^{1 / 6}
$$

where "days" is the number of exposure days per year, $n$ is the number of years of exposure, $c$ is a constant representing the static stress due to gravitational force (a value of $c=$ $0.25 \mathrm{MPa}$ can be normally used for driving posture), $S_{u i}$ is the ultimate strength of the lumbar spine for a person of age $(b+i)$ years, with $b$ being the age at which the exposure starts. The value $S_{u i}$ varies with the bone density of the vertebrae, which normally is reduced with age. From invitro studies ${ }^{10,12)}$, the following relationship between $S_{u i}$ (in $\mathrm{MPa}$ ) and $b+i$ (in years) has been derived: $S_{u i}=6.75$ $0.066(b+i)$. 